\title{
Online tool to assist volunteer firefighters in Santa Catarina State
}

\author{
Igor Benedet \\ Departamento de Informática \\ Instituto Federal Catarinense \\ Camboriú SC, Brazil \\ benedetigor@gmail.com
}

\author{
Daniel de Andrade Varela \\ Departamento de Informática \\ Institution/University Name \\ City State Country \\ daniel.varela@ifc.edu.br
}

\author{
Rodrigo Ramos Nogueira \\ Dep de Engenharia e Informática \\ Universidade de Coimbra \\ Coimbra, Portugal \\ rodrigonogueira@dei.uc.pt
}

\begin{abstract}
With a commitment to protect and guarantee the safety of the residents of Joinville, 127 years ago a group of people created an institution focused on serving the population in a fast and efficient way. This was how the Volunteer Fire Brigade of Joinville was born, the first such institution in Brazil and the second national fire company. Having seen that it is an association composed of several institutions, as well as several volunteers, a way of managing them is necessary. This paper presents the steps of developing an application to assist the ABVESC firefighters with regard to their institutional organization, bringing greater accessibility and agility to their tasks and planning.
\end{abstract}

\section{KEYWORDS}

Acessibility, Assistance, Integration, Technology;

\section{Introdução}

Os bombeiros voluntários são pessoas que dedicam parte do seu cotidiano para auxiliar o próximo, muitas vezes salvando vidas. A maioria dos bombeiros voluntários afirmam estar motivados por estarem sob controle naquilo que fazem e na forma como o fazem. A motivação mais possante, para o voluntariado nos bombeiros, era a ajuda da comunidade, seguindo-se o desejo de contribuir e de estar sobre controle. Outro dos fatores motivadores para o voluntariado nos bombeiros é o fato deste tipo de atividade ser uma oportunidade para combater o isolamento social. Os bombeiros são uma estrutura presente em todo lado, são eles que desencadeiam a organização e a identidade das comunidades [1]. A ABVESC (Associação de Bombeiros Voluntários do Estado de Santa Catarina) é uma associação que traz consigo 25 anos de história. Foi em 4 de abril de 1994 que Arthur Zietz e José Henrique Loyola tiveram a ideia de unir as corporações já existentes de bombeiros voluntários do Estado de Santa Catarina, e que atualmente, conta com 31 corporações e 4.600 pessoas, divididas em 50 municípios do Estado. Só em 2018 foram efetuados cerca de 76.585 chamados.

In: XVI Workshop de Trabalhos de Iniciação Científica (WTIC 2019), Rio de Janeiro, Brasil. Anais Estendidos do Simpósio Brasileiro de Sistemas Multimídia e Web (WebMedia). Porto Alegre: Sociedade Brasileira de Computação, 2019.

(C) 2019 SBC - Sociedade Brasileira de Computação.

ISSN: 2596-1683
Os bombeiros voluntários são pessoas que dedicam parte do seu cotidiano para auxiliar o próximo, muitas vezes salvando vidas. A maioria dos bombeiros voluntários afirmam estar motivados por estarem sob controle naquilo que fazem e na forma como o fazem. A motivação mais possante, para o voluntariado nos bombeiros, era a ajuda da comunidade, seguindo-se o desejo de contribuir e de estar sobre controle. Outro dos fatores motivadores para o voluntariado nos bombeiros é o fato deste tipo de atividade ser uma oportunidade para combater o isolamento social. Os bombeiros são uma estrutura presente em todo lado, são eles que desencadeiam a organização e a identidade das comunidades [1]. A ABVESC (Associação de Bombeiros Voluntários do Estado de Santa Catarina) é uma associação que traz consigo 25 anos de história. Foi em 4 de abril de 1994 que Arthur Zietz e José Henrique Loyola tiveram a ideia de unir as corporações já existentes de bombeiros voluntários do Estado de Santa Catarina, e que atualmente, conta com 31 corporações e 4.600 pessoas, divididas em 50 municípios do Estado. Só em 2018 foram efetuados cerca de 76.585 chamados.

A prestação dos serviços pelos bombeiros voluntários é essencial para a população, principalmente no que se refere à prestação de serviços em prol da vida. Torna-se necessário então, destacar a grande dificuldade que é assegurar a coesão de um número significativo de corporações que se encontram em cidades diferentes. A padronização das técnicas e procedimentos necessários aos membros da ABVESC é o principal fator que indica a necessidade de se ter uma plataforma em que todos tenham acesso e possam se organizar facilmente.

Tendo em vista esta problemática, foi proposto o desenvolvimento de uma aplicação que tem como objetivo facilitar e agilizar o processo de integração destas corporações, desenvolvendo um ambiente em que as novas instruções normativas dos bombeiros voluntários possam ser acessadas e baixadas facilmente, assim como o voto possa ser realizado de maneira ágil dentro do aplicativo, para facilitar o trabalho como um todo da referida instituição.

O aplicativo terá como funcionalidade principal armazenar as instruções normativas dos bombeiros, que precisam ser aprovadas e votadas pelos membros da ABVESC. Estas normativas ainda não possuem um sistema que automatize esse processo, sendo este, um dos principais objetivos do trabalho. O aplicativo também será disponibilizado para toda a comunidade, assim será possível acompanhar as principais notícias dos bombeiros e saber um 
pouco mais sobre sua história e missão. O app será desenvolvido usando duas frameworks principais, Apache Cordova e NodeJS. É esperado que o sistema ajude os bombeiros voluntários no que se refere a sua organização de forma eficiente e ágil, garantindo um melhor aproveitamento do tempo de todos os membros da instituição, assim como possibilite a coesão de todas as corporações do Estado de Santa Catarina.

\section{Trabalhos Relacionados}

Este artigo apresenta o desenvolvimento de uma aplicação híbrida para auxílio no gerenciamento de um grupo de bombeiros voluntários. Durante a fase de levantamento bibliográfico foram encontradas aplicações similares que serviram de base de conhecimento para o desenvolvimento.

Um exemplo foi o projeto que apresenta o desenvolvimento de um aplicativo para auxílio ao atendimento de ocorrências realizadas pela associação humanitária de busca, salvamento aéreo e resgate [10]. Neste projeto os autores apresentam uma aplicação com o objetivo de agilizar o atendimento dos bombeiros da cidade de Guaramirim no estado de Santa Catarina. A partir da automatização do preenchimento da ficha de atendimento dos bombeiros, que será feita via aplicativo.

A aplicação FIRECAST [11], um aplicativo para auxílio no atendimento a emergências no âmbito do corpo de bombeiros militar de Santa Catarina. Os autores apresentam que é a proposta de desenvolvimento de uma aplicação com o objetivo de gerenciar ocorrências dos bombeiros militares em determinada cidade. $\mathrm{O}$ aplicativo foi disponibilizado para o corpo de bombeiros e também para a comunidade.

\section{Metodologia}

No que se refere à categoria, este trabalho é uma pesquisa aplicada [2], pois teve seu início com a elaboração de um repertório conceitual e fundamentação teórica, trabalhos relacionados e estado da arte. Para isto foi realizada pesquisa exploratória, bibliográfica e documental, através de artigos, livros e reportagens on-line. Como se trata de um aplicativo, sendo assim um produto de tecnologia, esta pesquisa de enquadra como tecnológica [3], e os passos do desenvolvimento são descritos a seguir. .

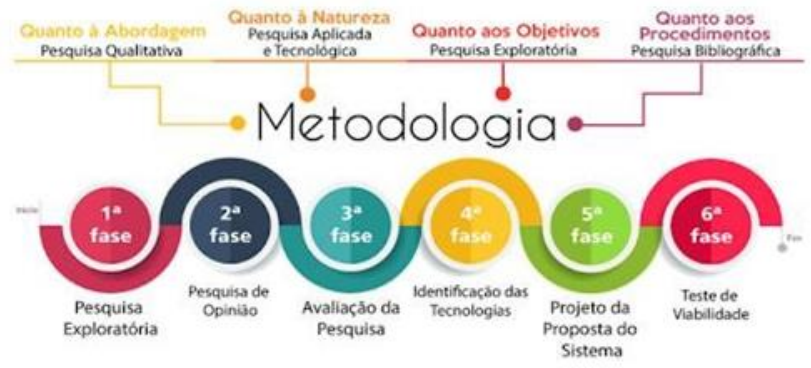

Figura 1: Metodologia [4]

O projeto teve início com a etapa de modelagem, a partir do levantamento de requisitos, seguido da delimitação do seu escopo, bem como perfil de usuários. Foi realizada a modelagem da base de dados do sistema e, por fim, o desenvolvimento dos protótipos da interface do aplicativo. Durante o processo de modelagem do sistema buscou-se adaptar sua usabilidade para o conhecimento e experiência do usuário, considerando a interação entre bombeiros voluntários e instituições.

Sobre o desenvolvimento da aplicação, trata-se de uma aplicação híbrida, tipo que agrega as tecnologias de desenvolvimento e programação de aplicações nativas e Web com as linguagens de marcação de hipertexto HTML[5], CSS[6] e Javascript, utilizandose do editor de texto Visual Studio Code[7]. Para implementação do banco de dados, será utilizado o sistema gerenciador de banco de dados MariaDB, utilizando a ferramenta de administração Dbeaver[8]. Para desenvolvimento do backend, como por exemplo, login/cadastro, download de pdfs das normas, votos, será utilizado a linguagem de programação NodeJs. As páginas Web serão convertidas em um aplicativo mobile com o uso do framework Apache Cordova [9].

\section{Resultados Parciais}

A Tabela 1 apresenta os requisitos listados para que o software se torne viável e possa atender as necessidades do cliente em relação ao app.

Tabela 1: Requisitos

\begin{tabular}{|c|c|c|}
\hline Requisito & Descrição & Categoria \\
\hline RNF1 & $\begin{array}{l}\text { O banco utilizado será o } \\
\text { MariaDB/MySql e a } \\
\text { linguagem para integração } \\
\text { com o software deverá ser } \\
\text { Javascript, via Nodejs }\end{array}$ & Sistema \\
\hline RNF2 & $\begin{array}{l}\text { A base de dados deve ser } \\
\text { protegida para acesso de } \\
\text { apenas usuários autorizados }\end{array}$ & Segurança \\
\hline RF1 & $\begin{array}{l}\text { Apenas usuários autorizados } \\
\text { (pelo menos um de cada } \\
\text { corporação) podem cadastrar } \\
\text { instruções normativas e } \\
\text { eventos }\end{array}$ & Segurança \\
\hline RF2 & $\begin{array}{l}\text { Apenas usuários que } \\
\text { possuem login e senha } \\
\text { podem efetuar o download de } \\
\text { instruções normativas }\end{array}$ & Segurança \\
\hline RF3 & $\begin{array}{l}\text { O app deve disponibilizar as } \\
\text { notícias da ABVESC para } \\
\text { todos os usuários }\end{array}$ & Acessibilidade \\
\hline RF4 & $\begin{array}{l}\text { Apenas um representante de } \\
\text { cada corporação poderá } \\
\text { efetuar o voto sobre uma } \\
\text { normativa em votação }\end{array}$ & Interface \\
\hline RNF3 & $\begin{array}{l}\text { O software deve ser } \\
\text { compatível com sistema } \\
\text { android e ios }\end{array}$ & Portabilidade \\
\hline
\end{tabular}


Online tool to assist volunteer firefighters in Santa Catarina State

Até o momento o aplicativo se encontra em fase de testes, com a grande parte das telas e a implementação do banco de dados já finalizada. Na Figura 1 (B) apresenta-se a tela inicial do aplicativo onde será possível ler as notícias da ABVESC. Ao lado na Figura 1 (A) está a tela de login.

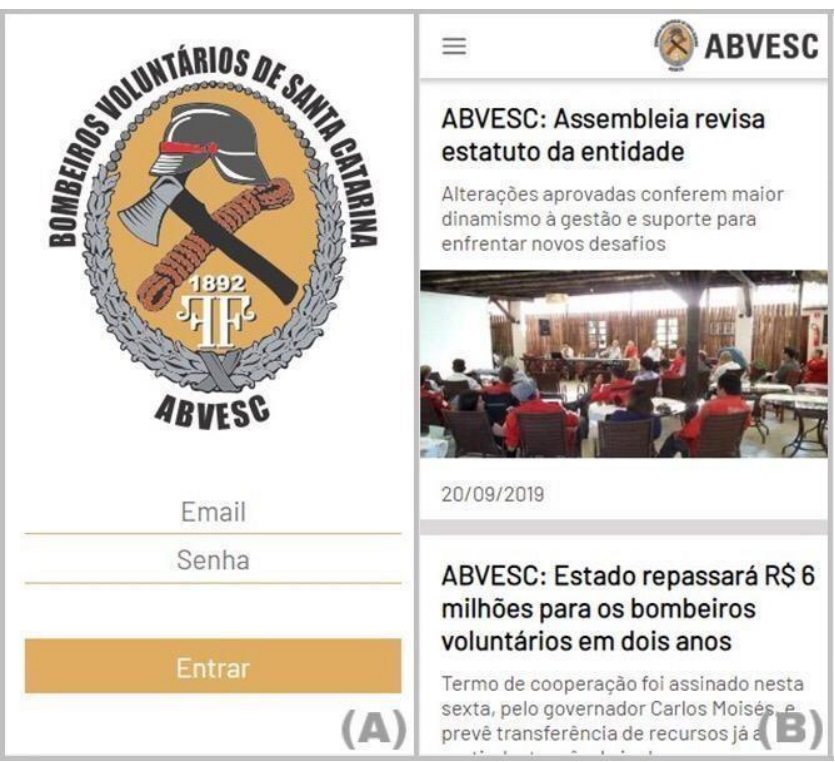

Figura 2: Em (A) tela de login, em (B) tela de notícias.

A Figura 2 (B) demonstra a tela onde será possível cadastrar uma nova instrução normativa no sistema. A Figura 2 (A) apresenta a tela que irá aparecer ao usuário após efetuar o login, onde será possível efetuar o download da apostila do curso e fazer o download das demais instruções normativas.

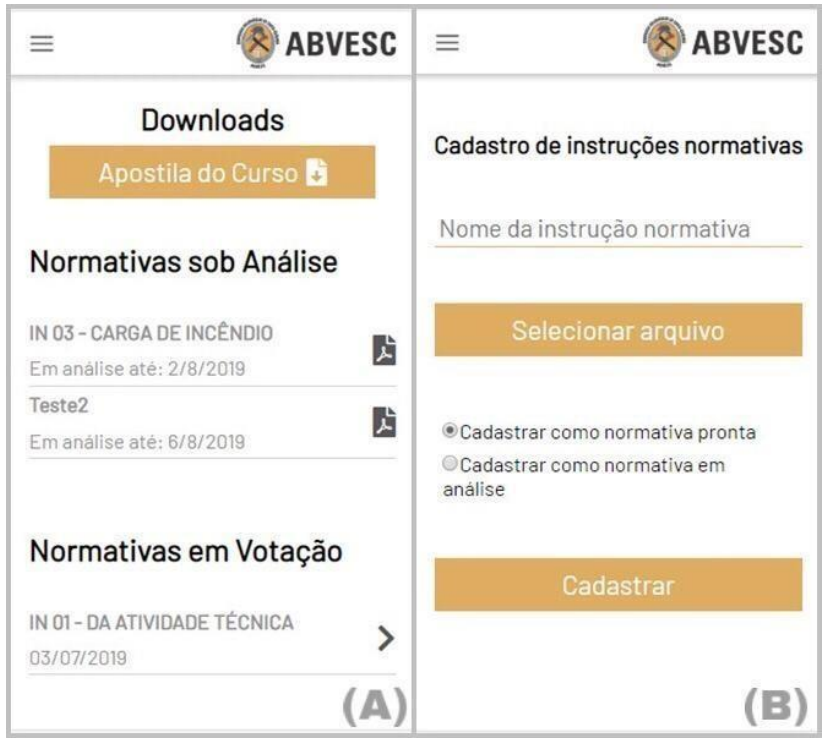

Figura 3. Em (A)Tela de download 1 e em (B) tela de cadastro de normativas.
Anais Estendidos do WebMedia'2019, Rio de Janeiro, Brasil

A Figura 3 (A) apresenta o menu lateral do aplicativo e a figura 2 (B) demonstra a segunda parte da tela de downloads

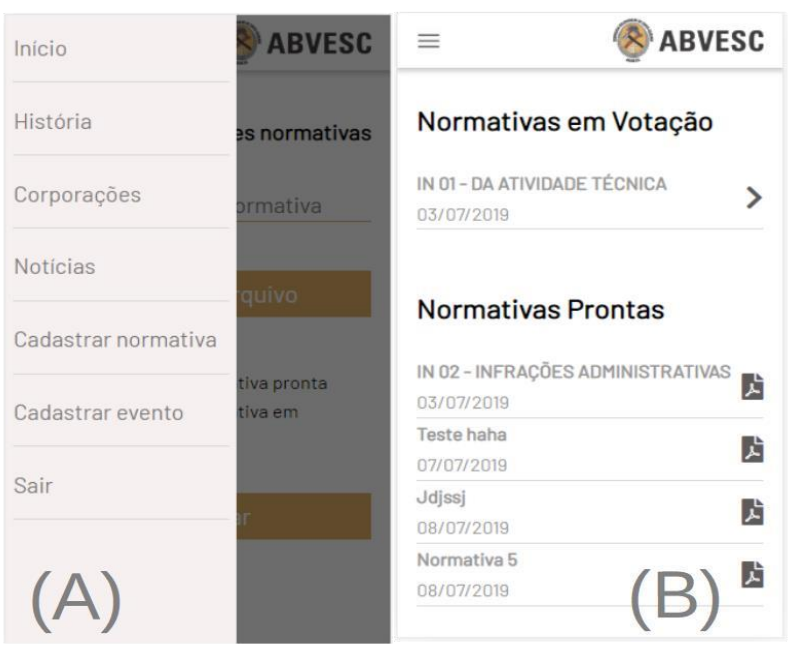

Figura 4. Em (A) menu lateral do aplicativo, em (B) parte final da tela de download. .

A Figura 2 (B) demonstra a tela onde será possível cadastrar uma nova instrução normativa no sistema. A Figura 2 (A) apresenta a tela que irá aparecer ao usuário após efetuar o login, onde será possível efetuar o download da apostila do curso e fazer o download das demais instruções normativas.

\section{Considerações Finais}

Levando em conta a importância para a sociedade que a ABVESC carrega consigo, é imprescindível que tal instituição tenha uma maneira ágil de organização. Este é o objetivo maior deste projeto, que busca ajudar de forma consciente uma instituição que atua em prol da vida, e fornece um importante apoio para a sociedade. É importante ressaltar que a aplicação proposta já se encontra em estado final de desenvolvimento. Na próxima etapa o aplicativo será disponibilizado para o teste real com os bombeiros voluntários, onde serão relatados todo e qualquer defeito que o sistema possa apresentar.

\section{REFERENCIAS}

[1] Thompson, A., \& Bono, B. (1993). Work without wages: The motivation for volunteer firefighters. The American Journal of Economics and Sociology, 323343.

[2] LAKATOS, E. Maria; MARCONI, Marina. de Andrade. Fundamentos de metodologia científica: Técnicas de pesquisa, v. 7, 2010.

[3] JUNIOR, Vanderlei FREITAS et al. A pesquisa científica e tecnológica. Espacios, v. 35, n. 9, 2014.

[4] TOZZI, Tatiana; ANDERLE, Daniel Fernando; NOGUEIRA, Rodrigo Ramos. Levantamento de tecnologias para ONGs de Proteção Animal para apoio ao resgate de animais domésticos acoplados ao ciclo de vida de um Sistema Web. In: Anais Estendidos do XXIV Simpósio Brasileiro de Sistemas Multimídia e Web. SBC, 2018. p. 81-84.

[5] MAKEUSEOF. HTML5. What Is HTML5, And How Does It Change The Way I Browse?. Disponível em: \&lt;http://www.makeuseof.com/tag/what-is- 
html5and-how-does-it-change-the-way-i-browse-makeuseof-explains/\&gt;. Acesso em: 10 out. 2016.

[6] MOZILLA DEVELOPER NETWORK. CSS. Disponível em: \&lt;https://developer.mozilla.org/pt-BR/docs/Web/CSS\&gt; Acesso em: 3 jul. 2019

[7] VISUAL Studio Code. 29 abr. 2015. Disponível em: https://code.visualstudio.com/. Acesso em: 3 jul. 2019.

[8] DBEAVER COMMUNITY. Disponível em: https://dbeaver.io/about/. Acesso em: 21 jul. 2019

[9] APACHE. Cordova. Apache Cordova: Mobile apps with HTML, CSS \&amp; JS Target multiple platforms with one code base Free and open source. Disponível em: \&lt;https://cordova.apache.org/\&gt;. Acesso em: 03 jul. 2019.

[10] Aplicativo para auxílio ao atendimento de ocorrências realizadas pela associação humanitária de busca, salvamento aéreo e resgate (AHBSAR), 2019. Disponível em: http://sinodalsuperior.ielusc.br/index.php/congresupsinodal20 19/article/view36>. Acesso em: 19 de jul. de 2019

[11] FIRECAST:APLICATIVO PARA AUXÍLIO NO ATENDIMENTO A EMERGÊNCIAS NO ÂMBITODO CORPO DE BOMBEIROS MILITAR DESANTA CATARINA, 2017. Disponível em: <http://150.162.242.35/handle/123456789/181423>. Acesso em: 19 de jul. de 2019

[12] MOZILLA DEVELOPER NETWORK. CSS. Disponível em: \&lt;https://developer.mozilla.org/pt-BR/docs/Web/CSS\&gt;.Acesso em: 3 jul. 2019.

[13] VISUAL Studio Code. 29 abr. 2015. Disponível em: https://code.visualstudio.com/. Acesso em: 3 jul. 2019.

[14] APACHE. Cordova. Apache Cordova: Mobile apps with HTML, CSS \&amp; JS Target multiple platforms with one code base Free and open source. Disponivel em: \&lt;https://cordova.apache.org/\&gt;. Acesso em: 03 jul. 2019. 